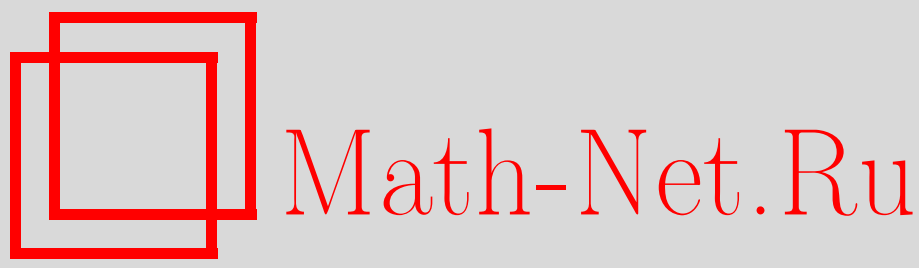

В. Е. Зотеев, Исследование и сравнительный анализ эффективности методов вычисления среднеквадратичных оценок коэффициентов линейно-параметрических дискретных моделей колебаний систем с турбулентным трением, Вестн. Сам. гос. техн. ун-та. Сер. Физ.-мат. науки, 2005, выпуск 34, 137-140

DOI: https://doi.org/10.14498/vsgtu347

Использование Общероссийского математического портала Math-Net.Ru подразумевает, что вы прочитали и согласны с пользовательским соглашением http://www . mathnet.ru/rus/agreement

Параметры загрузки:

IP: 54.210 .77 .194

26 апреля 2023 г., 12:39:19

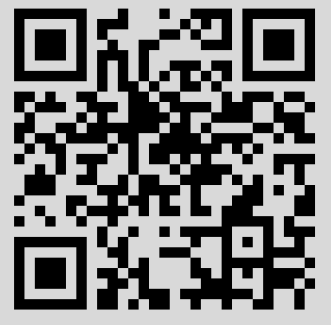




\section{ИССЛЕДОВАНИЕ И СРАВНИТЕЛЬНЫЙ АНАЛИЗ ЭФФЕКТИВНОСТИ МЕТОДОВ ВЫЧИСЛЕНИЯ СРЕДНЕКВАДРАТИЧНЫХ ОЦЕНОК КОЭФФИЦИЕНТОВ ЛИНЕЙНО-ПАРАМЕТРИЧЕСКИХ ДИСКРЕТНЫХ МОДЕЛЕЙ КОЛЕБАНИЙ СИСТЕМ С ТУРБУЛЕНТНЫМ ТРЕНИЕМ}

Исследуется эффективность двух численных методов определения среднеквадратичных оиенок коэффициентов линейно-параметрической дискретной модели колебаний систем с турбулентным трением. В основе методов лежат различные стохастические модели случайной аддитивной помехи и соответствующие преобразования линейно-параметрической дискретной модели, используемые в итерационных процедурах вычисления динамических характеристик систем с турбулентным трением.

Одним из эффективных методов определения динамических характеристик (ДХ) систем с турбулентным трением является метод, в основе которого лежат линейно-параметрические дискретные модели (ЛПДМ) свободных колебаний диссипативной системы [1]:

$$
y_{k}+y_{k-2}=\lambda_{0} y_{k-1}+\lambda_{1}\left[k y_{k}+(k-2) y_{k-2}\right]+\lambda_{2}(k-1) y_{k-1}+\eta_{k}, \quad k=\overline{2, N-1} .
$$

Коэффициенты ЛПДМ (1) связаны с ДХ системы декрементом $\delta_{0}$ и частотой $\omega$ колебаний соотношениями $\lambda_{0}=2 \cos \omega \tau, \lambda_{1}=-\frac{\delta_{0} \omega \tau}{2 \pi}$, где $\tau-$ период дискретизации экспериментальной виброграммы, $N$ - объем выборки результатов наблюдений $y_{k}$. Отсчеты $y_{k}$ содержат случайную аддитивную помеху $\varepsilon_{k}$, имеющую нормальное распределение, нулевое математическое ожидание, значения которой не коррелированны и имеют одинаковые дисперсии $\sigma_{\varepsilon}^{2}$. Очевидно, что при этом случайное возмущение $\eta_{k}$ в модели (1) может быть представлено в виде

$$
\eta_{k}=\varepsilon_{k}+\varepsilon_{k-2}-\lambda_{0} \varepsilon_{k-1}-\lambda_{1}\left[k \varepsilon_{k}+(k-2) \varepsilon_{k-2}\right]-\lambda_{2}(k-1) \varepsilon_{k-1}, \quad k=\overline{2, N-1} .
$$

Вычисление среднеквадратичных оценок коэффициентов модели (1) предполагает минимизацию функционала

$$
J(\lambda)=\sum_{k=2}^{N}\left[y_{k}+y_{k-2}-\lambda_{0} y_{k-1}-\lambda_{1}\left[k y_{k}+(k-2) y_{k-2}\right]-\lambda_{2}(k-1) y_{k-1}\right]^{2}=\sum_{k=2}^{N} \eta_{k}^{2} \Rightarrow \min
$$

на множестве $\left\{\lambda_{0}, \lambda_{1}, \lambda_{2}\right\}$. Однако полученные таким образом оценки будут асимптотически смещенными из-за корреляции между элементами $y_{k}$ и $\eta_{k}$ [2], что существенно увеличивает погрешность вычисления ДХ системы.

В $[2,3]$ рассматриваются методы среднеквадратичного оценивания параметров, устраняющие указанный недостаток. В их основе лежат авторегрессионные (АР) модели случайного возмущения $\eta_{k}$ [4]. Одним из таких методов является метод преобразования модели [2]. Рассмотрим эффективность применения этого метода при вычислении коэффициентов ЛПДМ (1).

Представим ЛПДМ (1) в виде обобщенной регрессионной модели

$$
f_{0 k}=\lambda_{0} f_{1 k}+\lambda_{1} f_{2 k}+\lambda_{2} f_{3 k}+\eta_{k},
$$

где $f_{0 k}=y_{k+2}+y_{k}, f_{1 k}=y_{k+1}, f_{2 k}=\left[k y_{k}+(k+2) y_{k+2}\right], f_{3 k}=(k+1) y_{k+1}, k=\overline{1, N-2}$. Для преобразования модели (3) случайное возмущение $\eta_{k}$ представим как авторегрессионный процесс порядка $s$ :

$$
\eta_{k}=\rho_{1} \eta_{k-1}+\rho_{2} \eta_{k-2}+\ldots+\rho_{s} \eta_{k-s}+\xi_{k}, \quad k=\overline{s+1, N-2},
$$

где $\xi_{k}$ - случайная величина, имеющая нормальное распределение, нулевое математическое ожидание, значения которой не коррелированны и имеют одинаковые дисперсии $\sigma_{\xi}^{2}$.

Вводя линейный оператор сдвига на $j$ шагов назад $z^{-j}\left\{y_{k}\right\}=y_{k-j}$, обобщенную регрессионную модель (3) можно преобразовать к виду

$$
f_{0 k}^{*}-\lambda_{0} f_{1 k}^{*}-\lambda_{1} f_{2 k}^{*}-\lambda_{2} f_{3 k}^{*}=\xi_{k}, k=\overline{1, N-2-s},
$$

где обобщенные регрессоры описываются формулами 


$$
f_{j k}^{*}=\left(1-\rho_{1} z^{-1}-\rho_{2} z^{-2}-\ldots-\rho_{s} z^{-s}\right) f_{j k}=f_{j k}-\sum_{q=1}^{s} \rho_{q} f_{j, k-q}, \quad j=\overline{0,3} .
$$

Применение классического метода наименьших квадратов к модели (6):

$$
\sum_{k=1}^{N-2-s}\left(f_{0 k}^{*}-\lambda_{0} f_{1 k}^{*}-\lambda_{1} f_{2 k}^{*}-\lambda_{2} f_{3 k}^{*}\right)^{2}=\sum_{k=1}^{N} \xi_{k}^{2} \Rightarrow \min
$$

позволяет получить наилучшие линейные оценки для коэффициентов $\lambda_{j}$ и тем самым повысить точность вычисления ДХ.

Алгоритм вычислений оценок коэффициентов ЛПДМ на основе преобразований, использующих АР модель случайной помехи, описывается следующей итерационной процедурой.

1. Обычным методом наименьших квадратов находятся начальные оценки $\lambda_{j}^{0}, j=\overline{1,3}$, для модели (3);

2. Вычисляются остатки для модели (3):

$$
e_{k}^{i}=f_{0 k}^{i}-\lambda_{0}^{i} f_{1 k}^{i}-\lambda_{1}^{i} f_{2 k}^{i}-\lambda_{2}^{i} f_{3 k}^{i}, \quad k=\overline{1, N-2} ;
$$

3. После подстановки в АР модель (4) вместо неизвестных значений случайной помехи $\eta_{k}$ остатков $e_{k}^{i}$, находятся оценки $\rho_{j}^{i}$ коэффициентов АР модели. Для этого минимизируется на множестве $\left\{\rho_{j}^{i}\right\}, j=\overline{1, s}$, функционал $J(\rho)=\sum_{k=1}^{N-2-s}\left(e_{k}^{i}-\sum_{j=1}^{s} \rho_{j}^{i} e_{k-j}^{i}\right)^{2} \Rightarrow \min$;

4. По формулам $f_{j k}^{i+1}=f_{j, k+s}^{i}-\sum_{q=1}^{s} \rho_{q}^{i} f_{j, k+s-q}^{i}, k=\overline{1, N-2-s(i+1)}, j=\overline{0,4}$ преобразуются регрессоры обобщенной модели к виду (6);

5. Методом наименьших квадратов находятся оценки $\lambda_{j}^{i+1}$ для преобразованной модели (5).

Этапы 2 - 5 выполняют итеративно, вычисляются на каждом $i$ - том этапе $(i=0,1,2, \ldots)$ остатки $e_{k}^{i}$, а затем оценки коэффициентов ЛПДМ $\lambda_{j}^{i+1}$. Процедура заканчивается после достижения заданного числа итераций или по критерию суммы квадратов остатков (7). В [2] утверждается, что для простых примеров этот метод дает хорошие результаты при разумно выбранном порядке объекта.

Проведены численно-аналитические исследования эффективности описанного метода преобразования модели на основе $\mathrm{AP}(s)$ модели (4) при вычислении коэффициентов линейно параметрической модели колебаний систем с турбулентным трением. Генерировалась выборка значений функции $y(t)=a_{0}\left(1+\frac{\delta_{0} \omega}{2 \pi} t\right)^{-1} \cos \left(\omega t+\psi_{0}\right)$, описывающей свободные колебания систем с турбулентным трением, с периодом дискретизации $\tau=0,2 T$ и объемом $N=50$. Параметры тестового сигнала имели следующие значения: $a_{0}=1, \psi_{0}=1, \delta_{0}=0,05, \omega=2 \pi$. К отсчетам смоделированного сигнала добавлялась случайная аддитивная помеха мощностью $1 \%$ от основного сигнала. Результаты вычислений относительной погрешности для декремента колебаний усреднялись по 1000 независимых испытаний в одной точке численного эксперимента. В качестве модели случайного возмущения $\eta_{k}$ использовалась авторегрессионная модель (4), порядок которой $s$ изменялся от двух до шести. Число итераций в алгоритме вычислений равнялось шести. Относительная погрешность вычисления декремента колебаний при применении классического метода наименьших квадратов составила 28\%. Результаты исследований представлены на рис. 1. По ним можно сделать вывод, что увеличение порядка АР модели случайной помехи с 2 до 5 при трех-пяти итерациях позволяет уменьшить погрешность вычислений на порядок по сравнению с классическим методом наименьших квадратов. 

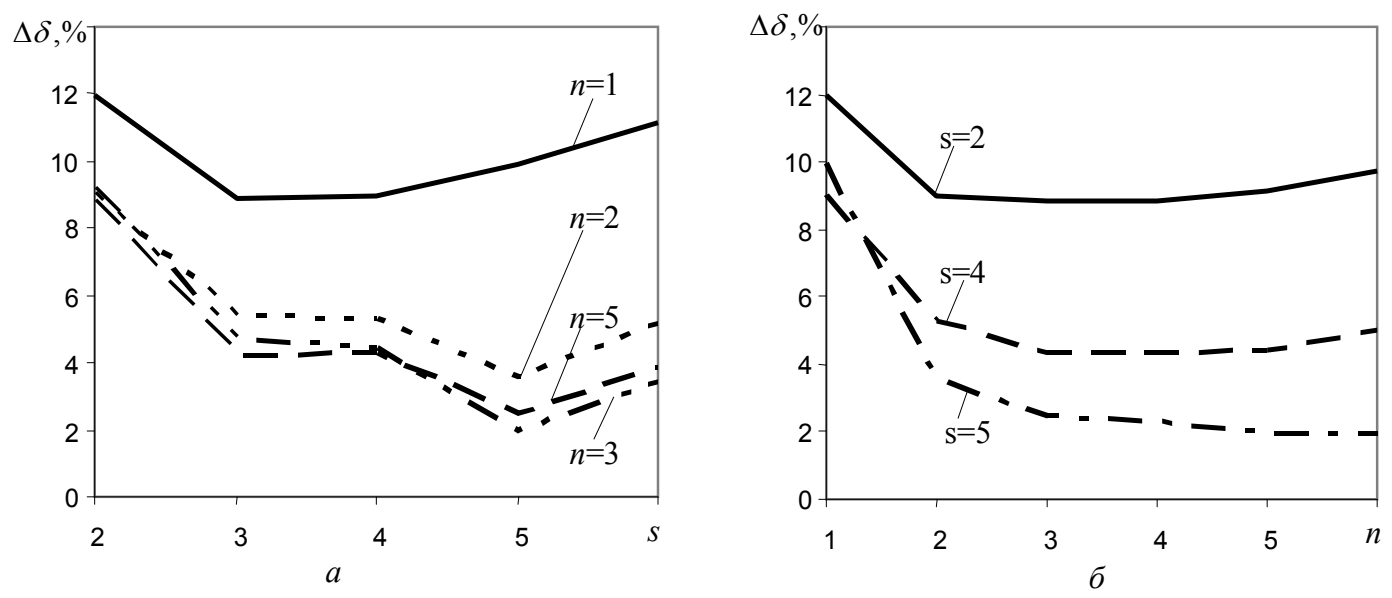

Р и с. 1. Зависимости относительной погрешности вычисления декремента колебаний от порядка $\mathrm{AP}(s)$ модели случайной помехи (а) и от числа итераций $n$ в ОМНК (б) при использовании авторегрессионой модели (4)

Применение рассмотренного выше метода преобразования модели при вычислении коэффициентов ЛПДМ имеет существенный недостаток: он не учитывает априорно известный характер зависимости $\eta_{k}$ от случайной аддитивной помехи $\varepsilon_{k}$ в результатах наблюдений. Для устранения этого недостатка предлагается итерационный численный метод, в основе которого также лежит преобразование ЛПДМ колебаний системы с турбулентным трением, но в качестве стохастической модели случайного возмущения $\eta_{k}$ используется модель (2). Эта модель относится к классу стохастических моделей скользящего среднего [4]. Возмущение $\eta_{k}$ можно представить в виде $\eta_{k}=\left(1-\lambda_{0} z^{-1}+z^{-2}\right)\left\{\left(1+k \lambda_{1}\right) \varepsilon_{k}\right\}$. Пусть существует обратный оператор $\left(1-\lambda_{0} z^{-1}+z^{-2}\right)^{-1}$. Тогда его можно представить в виде бесконечного ряда: $\left(1-\lambda_{0} z^{-1}+z^{-2}\right)^{-1}=1+\sum_{q=1}^{\infty} \alpha_{q} z^{-q}$, в котором коэффициенты $\alpha_{q}$ связаны рекуррентной формулой

$$
\alpha_{q}=\lambda_{0} \alpha_{q-1}-\alpha_{q-2}, q=2,3,4, \ldots, \alpha_{0}=1, \alpha_{1}=\lambda_{0} .
$$

С учетом этого из (2) можно получить

$$
\varepsilon_{k}=\frac{1}{1+k \lambda_{1}}\left(1-\lambda_{0} z^{-1}+z^{-2}\right)^{-1}\left\{\eta_{k}\right\}=\frac{1}{1+k \lambda_{1}}\left(\eta_{k}+\sum_{q=1}^{\infty} \alpha_{q} \eta_{k-q}\right) .
$$

Ограничиваясь в этом разложении $s$ первыми членами, получаем авторегрессионную модель $\mathrm{AP}(s)$ случайного возмущения $\eta_{k}$ порядка $s$ :

$$
\varepsilon_{k}=\frac{1}{1+k \lambda_{1}}\left(1+\sum_{q=1}^{s} \alpha_{q} z^{-q}\right) \eta_{k}=\sum_{q=0}^{s} \rho_{q} \eta_{k-q},
$$

в которой коэффициенты $\rho_{q}$ описываются формулами

$$
\rho_{q}=\frac{\alpha_{q}}{1+k \lambda_{1}}, \quad q=\overline{0, s} .
$$

В отличие от рассмотренного выше метода здесь коэффициенты АР модели (9) могут вычисляться не по остаткам $e_{k}$, а по найденным на предыдущей итерации коэффициентам ЛПДМ (1). Алгоритм вычислений оценок коэффициентов ЛПДМ на основе преобразований, использующих стохастическую модель (9) случайной помехи $\eta_{k}$, описывается следующей итерационной процедурой.

1. Обычным методом наименьших квадратов находятся начальные оценки $\lambda_{j}^{0}, j=\overline{1,3}$, для модели (3); 
2. По формулам (8) и (10) вычисляются коэффициенты $\rho_{q}^{i}$ для модели (9);

3. По формулам $f_{j k}^{i+1}=\sum_{q=0}^{s} \rho_{q}^{i} f_{j, k+s-q}^{i}, k=\overline{1, N-2-s(i+1)}, j=\overline{0,4}$, преобразуются регрессоры обобщенной модели (3);

4. Методом наименьших квадратов находятся оценки $\lambda_{j}^{i+1}$ для преобразованной модели (5).

Этапы 2 - 5 выполняются итеративно. Процедура заканчивается после достижения заданного числа итераций.

При тех же самых исходных данных и аналогичной организации численного эксперимента были проведены исследования эффективности применения метода преобразования ЛПДМ на основе стохастической модели случайного возмущения (2). Результаты исследований представлены на рис. 2.
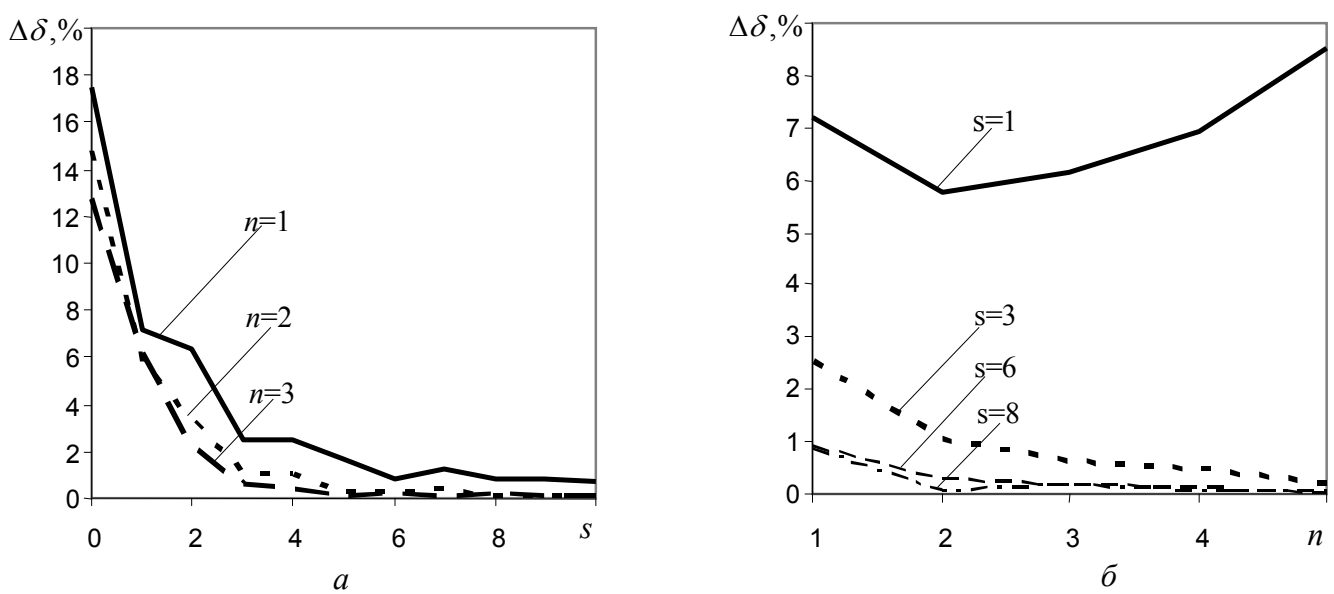

Р и с. 2. Зависимости относительной погрешности вычисления декремента колебаний от порядка $\mathrm{AP}(s)$ модели случайной помехи (а) и от числа итераций $n$ в ОМНК (б) при использовании стохастической модели (2)

По результатам этих исследований можно сделать вывод, что увеличение как порядка стохастической модели (9) случайного возмущения до 10 , так и числа итераций в алгоритме вычислений до пяти приводит к существенному уменьшению погрешности вычислений. В частности, относительная погрешность вычисления декремента колебаний уменьшилась с $28 \%$ при классическом методе наименьших квадратов до $0,1 \%$ при использовании метода преобразования ЛПДМ на основе стохастической модели случайного возмущения (2). При сравнении эффективности применения двух рассмотренных выше методов вычисления среднеквадратичных оценок коэффициентов ЛПДМ на основе преобразования обобщенной регрессионной модели очевидное преимущество имеет итерационный численный метод, использующий априорно известный вид стохастической модели случайного возмущения. Результаты вычислений с использованием этого метода на порядок точнее оценок, полученных методом, использующим классическую АР модель случайной помехи.

\section{БИБЛИОГРАФИЧЕСКИЙ СПИСОК}

1. Зотеев В.E. Разработка и исследование линейных дискретных моделей колебаний диссипативных систем // Вестн. Сам. гос. техн. ун-та. Сер.: Физ.-мат. науки, 1999. № 7 С. 170-177.

2. Вучков И., Бояджиева Л., Солаков Е. Прикладной линейный регрессионный анализ. М.: Финансы и статистика, 1987. $342 \mathrm{c}$.

3. Штейнберг Ш.Е. Идентификация в системах управления. М.: Энергоатомиздат, 1987. 80 с.

4. Бокс. Дж., Дженкинс Г. Анализ временных рядов. Прогноз и управление. Вып. 1. М.: Мир, 1974. 406 с. 